\title{
A Fast Algorithm of Generalized Radon-Fourier Transform for Weak Maneuvering Target Detection
}

\author{
Weijie Xia, Ying Zhou, Xue Jin, and Jianjiang Zhou \\ Key Laboratory of Radar Imaging and Microwave Photonics, Ministry of Education, College of Electronic and Information Engineering, \\ Nanjing University of Aeronautics and Astronautics, Nanjing 211100, China
}

Correspondence should be addressed to Weijie Xia; nuaaxwj@nuaa.edu.cn

Received 1 July 2016; Accepted 5 October 2016

Academic Editor: Ana Alejos

Copyright (C) 2016 Weijie Xia et al. This is an open access article distributed under the Creative Commons Attribution License, which permits unrestricted use, distribution, and reproduction in any medium, provided the original work is properly cited.

The generalized Radon-Fourier transform (GRFT) has been proposed to detect radar weak maneuvering targets by realizing coherent integration via jointly searching in motion parameter space. Two main drawbacks of GRFT are the heavy computational burden and the blind speed side lobes (BSSL) which will cause serious false alarms. The BSSL learning-based particle swarm optimization (BPSO) has been proposed before to reduce the computational burden of GRFT and solve the BSSL problem simultaneously. However, the BPSO suffers from an apparent loss in detection performance compared with GRFT. In this paper, a fast implementation algorithm of GRFT using the BSSL learning-based modified wind-driven optimization (BMWDO) is proposed. In the BMWDO, the BSSL learning procedure is also used to deal with the BSSL phenomenon. Besides, the MWDO adjusts the coefficients in WDO with Levy distribution and uniform distribution, and it outperforms PSO in a noisy environment. Compared with BPSO, the proposed method can achieve better detection performance with a similar computational cost. Several numerical experiments are also provided to demonstrate the effectiveness of the proposed method.

\section{Introduction}

With the development of aircraft stealth technology, there is a growing need for radar to detect weak maneuvering targets in a noisy background. It is a known fact that pulse integration especially coherent integration can improve the signal-tonoise ratio (SNR) and ultimately improve the detection performance [1].

Concentrating on coherent integration, a lot of work has been carried out. The most commonly used method is moving target detection (MTD) [2], which achieves integration by using Doppler filter bank. However, MTD method can only deal with the target with uniform velocity and will become invalid if the range migration (RM) exceeds one range bin during the integration time [3]. It is of vital importance to eliminate RM since the high-speed target can easily exceed several range units even in a short time. To deal with RM, keystone transform (KT) [4,5] was performed by rescaling the time axis for each frequency and is often performed before MTD. In actual detecting environment, for example, the velocity, acceleration, and jerk will result in first-order RM, second-order RM, and third-order RM, respectively. Unfortunately, conventional KT can only correct the first-order RM. Thus [6-8] studied second-order KT to correct the second-order RM and Kong et al. [9] proposed a coherent integration method via generalized KT and generalized dechirp process (GKTGDP) for maneuvering targets with arbitrary high-order RM. It is worth paying attention to that KT could be invalid without ambiguity correction if Doppler ambiguity happens. Algorithms for Doppler ambiguity correction are hardly independent of Doppler ambiguous integers searching; thus, the computational burden will greatly increase.

In recent years, a new method called Radon-Fourier transform (RFT) [10-12] has been proposed to realize longtime coherent integration via jointly searching along range and velocity directions. The detection performance of the high-speed and weak targets with constant velocity can be significantly improved by RFT if one of the searching pairs matches well with actual values. In consideration of maneuvering targets, generalized RFT (GRFT) [10, 13] was also defined for targets with arbitrary parameterized 
motion. Based on the idea of GRFT, a lot of work has been done recently [14-17]. Actually, the GRFT suffers from heavy computational burden and is impractical without fast implementations because of the multidimensional ergodic search. Fortunately, the realization of GRFT can be converted into an optimization problem in parameter space. Thus, intelligent optimization algorithms can be utilized to eliminate a large number of unnecessary searching paths. Another drawback of GRFT is the BSSL problem $[10,11]$ derived from discrete pulse sampling, finite range resolution, and limited integration time, which will lead to intelligent optimization algorithms converging to local optimum easily. Following consideration of the above issues, Qian et al. [18] have proposed BSSL learning-based particle swarm optimization (BPSO) to fast implement GRFT. By using the relation of BSSL and the main lobe, the local convergence can be avoided and the convergence speed can be accelerated simultaneously.

Although BPSO-based GRFT is efficient, it suffers from apparent detection performance loss compared with GRFT. To improve the detection performance, this paper proposes the BSSL learning-based modified wind-driven optimization (BMWDO). The wind-driven optimization (WDO) $[19,20]$ is a stochastic nature inspired, population based iterative heuristic global optimization method based on atmospheric motion. Compared with the traditional PSO, WDO employs additional terms in the velocity update equation, providing robustness and extra degrees of freedom for fine-tuning. However, it could be difficult in choosing optimum WDO coefficients for GRFT because the location of the optimum point depends on the motion parameters which have large dynamic ranges. In order to deal with the difficulty in choosing coefficients and further improve the global optimization ability of WDO in a noisy environment, we propose a modified WDO method which adjusts the control coefficients in WDO with random distributions, namely, MWDO. Detailed numerical experiments demonstrate that the proposed BMWDO method can improve the detection performance with a similar running time compared with BPSO.

\section{Signal Model and GRFT}

2.1. Signal Model. Suppose that radar transmits linear frequency modulated (LFM) signal, that is,

$$
s_{t}(\widehat{t})=\operatorname{rect}\left(\frac{\widehat{t}}{T_{p}}\right) \exp \left(j \pi \gamma \hat{t}^{2}\right) \exp \left(j 2 \pi f_{c} t\right),
$$

where

$$
\operatorname{rect}\left(\frac{\hat{t}}{T_{p}}\right)= \begin{cases}1, & \left|\frac{\hat{t}}{T_{p}}\right| \leq \frac{1}{2} \\ 0, & \left|\frac{\hat{t}}{T_{p}}\right|>\frac{1}{2},\end{cases}
$$

$T_{p}$ is the pulse width, $\gamma$ is the frequency modulated rate, and $f_{c}$ is the carrier frequency. Let $t_{m}=m T_{r}(m=0,2, \ldots, M-1)$ denote the slow time, where $T_{r}$ denotes the pulse repetition time, $M$ is the number of pulses, and $\hat{t}=t-m T_{r}$ is the fast time.
The received radar echo after carrier frequency demodulation can be denoted as

$$
\begin{aligned}
s_{r}\left(t_{m}, \widehat{t}\right)= & A_{0} \operatorname{rect}\left(\frac{\widehat{t}-2 R\left(t_{m}\right) / c}{T_{p}}\right) \\
& \cdot \exp \left[-j \frac{4 \pi f_{c} R\left(t_{m}\right)}{c}\right] \\
& \cdot \exp \left[j \pi \gamma\left(\widehat{t}-\frac{2 R\left(t_{m}\right)}{c}\right)^{2}\right],
\end{aligned}
$$

where $R\left(t_{m}\right)$ is the distance between radar and target at the radar line-of-sight and $A_{0}$ is the amplitude of the echo. The time delay of the echo is $\tau\left(t_{m}\right)=2 R\left(t_{m}\right) / c$, where $c$ is the speed of light. After pulse compression via using the baseband transmitted signal as the reference signal, that is,

$$
H(\widehat{t})=\operatorname{rect}\left(\frac{\widehat{t}}{T_{p}}\right) \exp \left(j \pi \gamma \widehat{t}^{2}\right),
$$

the received signal in the time domain can be expressed as

$$
\begin{aligned}
& S_{P C}\left(t_{m}, \widehat{t}\right) \\
& \quad=A_{1} \operatorname{sinc}\left(\pi B\left(\widehat{t}-\tau\left(t_{m}\right)\right)\right) \exp \left[-j 2 \pi f_{c} \tau\left(t_{m}\right)\right] .
\end{aligned}
$$

In the above equation, $A_{1}$ is the amplitude after pulse compression and $B$ denotes the system bandwidth. The range between radar and target in radial direction varies with the slow time $t_{m}$ and can usually be expressed as a polynomial function of $t_{m}$, which can be expanded into Taylor series [16], that is,

$$
R\left(t_{m}\right)=\sum_{p=0}^{P-1} \frac{1}{p !} \alpha_{p} t_{m}^{p}, \quad t_{m} \in\left[\frac{-T_{n}}{2}, \frac{T_{n}}{2}\right],
$$

where $P-1$ is the motion order, $T_{n}$ is the coherent integration time, and $\boldsymbol{\alpha}^{P}=\left[\alpha_{0}, \alpha_{1}, \ldots, \alpha_{P-1}\right]$ is the motion parameter vector. It is obvious that the peak location of the sinc function varies with $t_{m}$ and the changes will exceed the range resolution $\rho_{r}=c / 2 B$ easily if the integration time is long or the motion parameters are not very small, which means that the across range unit (ARU) effect will happen. The Doppler frequency can be calculated as

$$
f_{d}=\frac{2}{\lambda} \frac{d R\left(t_{m}\right)}{d t_{m}}=f_{0}+\frac{2}{\lambda} \sum_{p=2}^{P-1} \frac{1}{(p-1) !} \alpha_{p} t_{m}^{p-1}
$$

where $\lambda=c / f_{c}$ is the wavelength, $f_{0}=2 \alpha_{1} / \lambda$ is the central frequency of Doppler, and the parameter $\alpha_{1}$ is the target's velocity. If the target has acceleration or higher motion parameters, the Doppler frequency will be time-varying. If the changes of $f_{d}$ exceed the Doppler resolution $\rho_{d}=1 / T_{n}$, the Doppler frequency migration (DFM) will come across. In order to coherently accumulate the target's energy, we need to correct both the ARU and the DFM. 
2.2. Definition and Analysis of GRFT. GRFT is a coherent integration algorithm via jointly searching in multidimensional parameter space. By using GRFT, the trace of the target can be extracted and the DFM can be compensated at the same time. The definition of GRFT in [10] is given as follows.

Definition 1. Suppose a 2D complex function $f\left(t_{m}, \widehat{t}\right) \in C$ is defined in the $\left(t_{m}, \widehat{t}\right)$ plane and a parameterized $P$ dimensional function $\hat{t}=\eta\left(t_{m} ; \widehat{\boldsymbol{\alpha}}^{P}\right)$ is used for searching a certain time-varied curve in the plane, where $\widehat{\boldsymbol{\alpha}}^{P}=\left[\widehat{\alpha}_{0}\right.$, $\left.\widehat{\alpha}_{1}, \ldots, \widehat{\alpha}_{P-1}\right]$. Then GRFT can be defined as

$$
\begin{aligned}
G & \left(\widehat{\boldsymbol{\alpha}}^{P}\right) \\
& =\int_{-\infty}^{\infty} f\left(t_{m}, \eta\left(t_{m} ; \widehat{\boldsymbol{\alpha}}^{P}\right)\right) \exp \left(j 2 \pi \varepsilon \eta\left(t_{m} ; \widehat{\boldsymbol{\alpha}}^{P}\right)\right) d t_{m},
\end{aligned}
$$

where $\varepsilon$ is a known constant with respect to $\eta\left(t_{m} ; \widehat{\boldsymbol{\alpha}}^{P}\right)$.

$$
\begin{aligned}
& \text { Let } f\left(t_{m}, \widehat{t}\right)=S_{P C}\left(t_{m}, \widehat{t}\right) \text {; then (8) can be rewritten as } \\
& G\left(\widehat{\boldsymbol{\alpha}}^{P}\right)=\int_{-\infty}^{\infty} S_{P C}\left(t_{m}, \widehat{\tau}\left(t_{m}\right)\right) \exp \left(j 2 \pi f_{c} \widehat{\tau}\left(t_{m}\right)\right) d t_{m},
\end{aligned}
$$

where $\widehat{\tau}\left(t_{m}\right)=(2 / c) \sum_{p=0}^{P-1}(1 / p !) \widehat{\alpha}_{p} t_{m}^{p}$. Suppose that $P=2$; then GRFT degenerates into RFT which deals with the case of uniform velocity. From (9), we can easily know that when the searching values of motion parameters $\left[\widehat{\alpha}_{0}, \widehat{\alpha}_{1}, \ldots, \widehat{\alpha}_{P-1}\right]$ are exactly the target's real motion values $\left[\alpha_{0}, \alpha_{1}, \ldots, \alpha_{P-1}\right]$, the coherent integration could be achieved and the peak would be formed in the parameter space, that is,

$$
G\left(\boldsymbol{\alpha}^{P}\right)=M A_{1}
$$

Then the target can be detected and the motion parameters can be easily obtained by the location of the peak in the parameter space. However, because of limited integration time, discrete pulse sampling, and finite range resolution, the BSSL $[10,11]$ will also be formed in parameter space, which influences target detection performance. The causes of BSSL and the relations between BSSL and the main lobe are discussed as follows.

Equation (9) can be rewritten as

$$
\begin{gathered}
G\left(\widehat{\boldsymbol{\alpha}}^{P}\right)=\sum_{m=0}^{M-1} A_{1} \operatorname{sinc}\left(\pi B\left(\widehat{\tau}\left(t_{m}\right)-\tau\left(t_{m}\right)\right)\right) \\
\cdot \exp \left(j 2 \pi f_{c}\left(\widehat{\tau}\left(t_{m}\right)-\tau\left(t_{m}\right)\right)\right) \\
=\sum_{m=0}^{M-1} A_{1} \operatorname{sinc}\left(\pi B\left(\widehat{\tau}\left(t_{m}\right)-\tau\left(t_{m}\right)\right)\right) \\
\cdot \exp \left(j 4 \pi f_{c}\left(\widehat{\alpha}_{0}-\alpha_{0}\right)\right) \exp \left(\Delta \phi\left(t_{m}\right)\right),
\end{gathered}
$$

where

$$
\begin{aligned}
\Delta \phi & \left(t_{m}\right) \\
& =\frac{j 4 \pi f_{c}}{c\left[\left(\widehat{\alpha}_{1}-\alpha_{1}\right) t_{m}+\sum_{p=2}^{P-1}(1 / p !)\left(\widehat{\alpha}_{p}-\alpha_{p}\right) t_{m}^{p}\right]} .
\end{aligned}
$$

When $\widehat{\alpha}_{p}=\alpha_{p}(p=2, \ldots, P-1)$ and $\widehat{\alpha}_{1}-\alpha_{1}=q v_{b}$, where $v_{b}=c / 2 f_{c} T_{r}$ is the blind speed and $q$ is the blind speed integer, we have $\exp \left(\Delta \phi\left(t_{m}\right)\right)=1$ in (11). It is obvious that the phase can be compensated even though $q \neq 0$, which results in the BSSL phenomenon. Slice of BSSL can be denoted as

$$
\left|G\left(\widehat{\boldsymbol{\alpha}}^{P}\right)\right|=A_{1} \sum_{m=0}^{M-1} \operatorname{sinc}\left(\pi \frac{\widehat{\alpha}_{0}-\alpha_{0}+v_{b} q t_{m}}{\rho_{r}}\right) .
$$

The blind speed integer $q \in\left[\left(\widehat{\alpha}_{1 \min }-\alpha_{1}\right) / v_{b},\left(\widehat{\alpha}_{1 \max }-\alpha_{1}\right) / v_{b}\right]$, where $\left[\widehat{\alpha}_{1 \min }, \widehat{\alpha}_{1 \max }\right]$ is the searching range of velocity.

By analyzing (11), (12), and (13), we can see that the properties of BSSL are irrelevant to $\widehat{\alpha}_{p}(p=2, \ldots, P-1)$. Thus, the case of constant velocity is taken as an example to analyze the relations between BSSL and the main lobe. The sketch map of BSSL is illustrated in Figure 1. Suppose that the target's velocity is $v_{1}$, the initial range is $R_{0}$, and the one-time blind speed is $v_{2}=v_{1}+v_{b}$. The shadow region is formed so that the searching lines with one-time blind speed intersect the range-walk line in the integration time $\left[-T_{n} / 2, T_{n} / 2\right]$. The searching range of the shadow region is $\left[R_{0}-v_{b} T_{n} / 2, R_{0}+v_{b} T_{n} / 2\right] ;$ thus the length of the supporting area is

$$
L(1)=v_{b} T_{n}=\frac{c}{2 f_{c} T_{r}} \cdot M T_{r}=\frac{\lambda M}{2} .
$$

Because of the finite range resolution, the overlapped pulse number in the intersection of the range-walk line and the searching line is

$$
n=\frac{\rho_{r}}{v_{b} T_{r}}=\frac{2 \rho_{r}}{\lambda} .
$$

The $n$ pulses are also coherently integrated. Thus, the primary lobe to side lobe ratio (PSLR) can be denoted as

$$
\operatorname{PSLR}(1)=\frac{M}{n}=\frac{\lambda M}{2 \rho_{r}} .
$$

In general, in the case that the blind speed integer $q \neq 1$,

$$
\begin{aligned}
L(q) & =\frac{\lambda M|q|}{2}, \\
\operatorname{PSLR}(q) & =\frac{\lambda M|q|}{2 \rho_{r}} .
\end{aligned}
$$

With the increase of $|q|$, the supporting area of BSSL becomes longer and the amplitude of the side lobe decreases.

\section{Fast Implementation of GRFT via BMWDO}

3.1. Modified Wind-Driven Optimization. The wind-driven optimization (WDO) algorithm [19, 20] is inspired from the Earth's atmosphere where wind blows in an attempt to equalize imbalances in air pressure. The model of WDO is based on the definition of trajectories of small air parcels within the Earth atmosphere according to Newton's second law of motion. 


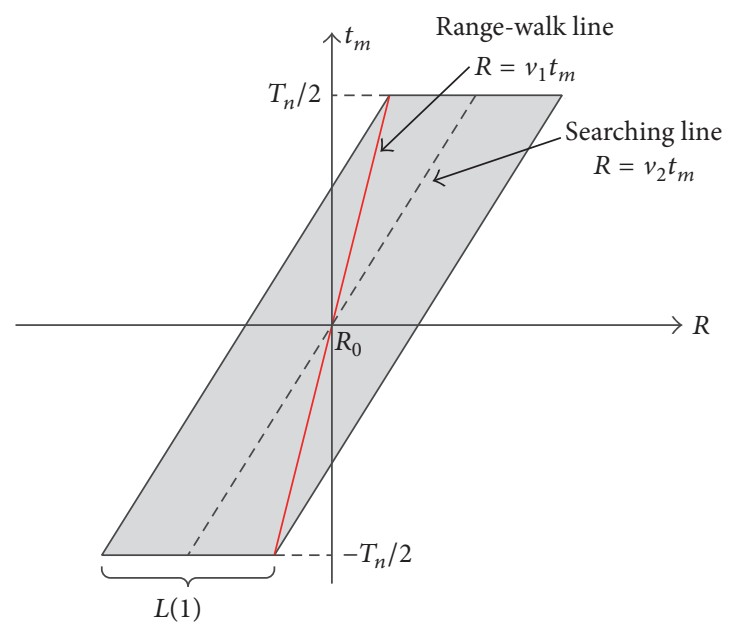

FIGURE 1: Sketch map of the BSSL phenomenon.

WDO is very similar to PSO [21] in that these air parcels are also described by positions and velocities which refer to candidate solutions and the amount of position displacement, respectively. Particles in WDO refer to small air parcels that are assumed dimensionless and weightless for simplification. In WDO, a population of air parcels is distributed throughout $N$-dimensional problem space, and the velocity of air parcel is updated in each iteration process based on the equation which is derived from Newton's second law of motion and the ideal gas laws. It is given by

$$
\begin{aligned}
\mathbf{U}_{\text {new }}= & (1-\alpha) \mathbf{U}_{\text {cur }}-g \mathbf{X}_{\text {cur }} \\
& +\left[R T\left|\frac{1}{i}-1\right|\left(\mathbf{X}_{\mathrm{opt}}-\mathbf{X}_{\text {cur }}\right)\right] \\
& +\left(\frac{C \mathbf{U}_{\text {cur }}^{\text {other dim }}}{i}\right),
\end{aligned}
$$

where $i$ represents the rankings of the air parcels since all the parcels are ranked according to their pressure. The "pressure" represents the value of the objective function in different problems. If we attempted to find the maximum (minimum) value of the objective function, parcels should be ranked in descending (ascending) order. Equation (18) demonstrates that the updated velocity $\mathbf{U}_{\text {new }}$ for the next iteration process is associated with the current velocity $\mathbf{U}_{\text {cur }}$, the current position $\mathbf{X}_{\text {cur }}$, the optimal position $\mathbf{X}_{\text {opt }}$ with the highest pressure value that has been found until the current iteration, and the current velocity $\mathbf{U}_{\text {cur }}^{\text {other dim }}$ which is randomly chosen from other dimensions. Coefficients $\alpha, g, R, T$, and $C$ are related to the friction coefficient, gravity, universal gas constant, temperature, and the influence of the Coriolis force in the physical model. The position of air parcel can be updated by

$$
\mathbf{X}_{\text {new }}=\mathbf{X}_{\text {cur }}+\left(\mathbf{U}_{\text {new }} \Delta t\right) \text {, }
$$

where $\Delta t=1$ is assumed for simplicity.

Before performing WDO, the four coefficients $\alpha, g, R T$, and $C$ should be chosen firstly. As illustrated in [19], the optimum performance of WDO can be achieved by selecting proper values for the four coefficients, but the optimum values of the WDO coefficients may vary from problem to problem. In GRFT, the optimum location of parcels is related to the target's motion state. In each detection process, the motion states of targets are difficult to predict and the motion parameters may have large dynamic ranges, so it is scarcely possible to find a single set of coefficients that will work efficiently in each case. Considering the problem, we propose the modified WDO (MWDO) to tune $\alpha, g, R T$, and $C$ in each iteration by random distributions. By applying MWDO, we can avoid a large number of trials to select the optimum coefficients. When choosing the appropriate random distribution, the global optimization ability of WDO in a noisy environment is primarily considered. The values of coefficients are given by

$$
\begin{aligned}
\alpha & =0.1 * \text { rand } L \\
R T & =0.1 * \text { rand } L, \\
g & =0.1 * \text { rand } L \\
C & =2.5 * \text { rand } \_U,
\end{aligned}
$$

where the random number rand_ $U$ is uniformly distributed between 0 and 1 and the random number rand $L$ is subject to Levy distribution. The Levy distribution $[22,23]$ is a continuous probability distribution for a nonnegative random variable and its probability density function over the domain $x \geq \mu$ is

$$
f(x ; \mu, \gamma)=\sqrt{\frac{\gamma}{2 \pi}} \frac{e^{-\gamma / 2(x-\mu)}}{(x-\mu)^{3 / 2}},
$$

where $\mu$ is the location parameter and $\gamma$ is the scale parameter. In (20), (21), and (22), $\mu=0$ and $\gamma=0.001$ are selected.

Through analyzing the characteristics of Levy distribution, we can find that the random number rand $L$ has a great 
probability to be very small values and will occasionally be big values. It means that, in most cases, other parcels move slowly to the current optimal parcel. In other words, the position updating step length is small but occasionally big. We know that the current optimal position is usually the noise peak position especially when the SNR is very low, so if other parcels move towards the current optimal one with a large step length, the global optimal solution will be missed with a great probability and the diversity of parcels will lose rapidly. The small step length of parcels helps to maintain the population diversity and reduce the probability of flying past the optimal solution. The occasional large step length avoids the parcels converging too slowly and helps parcels jump to other searching areas without being trapped in a small local area, which is useful in improving the global searching ability. Therefore, the Levy distribution is appropriate to tune the coefficients of WDO. By applying MWDO, the optimization ability of WDO in a noisy environment can be enhanced and, at the same time, the difficulty in choosing proper coefficients in WDO can be overcome.

3.2. BSSL Learning-Based MWDO in GRFT. When applying MWDO in GRFT, a great number of unnecessary searching paths can be eliminated, which means the GRFT can be calculated efficiently. However, big values of BSSL in GRFT may cause local convergence to side lobes. To settle this matter, we propose a BSSL learning-based MWDO to find the main lobe by using the relations between side lobes and the main lobe. The detailed description of the proposed method is given as follows and the whole target detection procedure based on BMWDO is shown in Figure 2.

\section{Step 1 (initialization).}

Step 1.1. Specify the basic conditions in MWDO, including the population size $S$, the maximum number of iterations $k_{\max }$, the dimension of the searching space $P$ which is related to the motion order, the searching range of each parameter, and the restrictions on velocities of air parcels. The location and velocity of each parcel can be denoted as $\mathbf{X}=\left[x_{1}, x_{2}, \ldots, x_{P}\right]$ and $\mathbf{U}=\left[u_{1}, u_{2}, \ldots, u_{P}\right]$, where $x_{1}, x_{2}, \ldots, x_{P}$ represents the searching motion parameter $\widehat{\alpha}_{0}, \widehat{\alpha}_{1}, \ldots, \widehat{\alpha}_{P-1}$, respectively.

Step 1.2. Initialize air parcels' locations by randomly distributing them in the searching space and initialize air parcels' velocities to 0 .

Step 1.3. Sort these parcels based on their pressure values. In GRFT, pressure value of parcel refers to the absolute value of GRFT: $|G(\mathbf{X})|$. The first parcel is the one which has the biggest pressure value and its location can be denoted as $\mathbf{X}_{\text {opt }}(0)$.

Step 2. Generate the values of coefficients of MWDO via (20), (21), (22), and (23). Then update the velocities and locations of air parcels based on (18) and (19), respectively. Sort these updated air parcels based on their pressure values and find the current optimal air parcel $\mathbf{X}_{\text {opt }}(k)$.

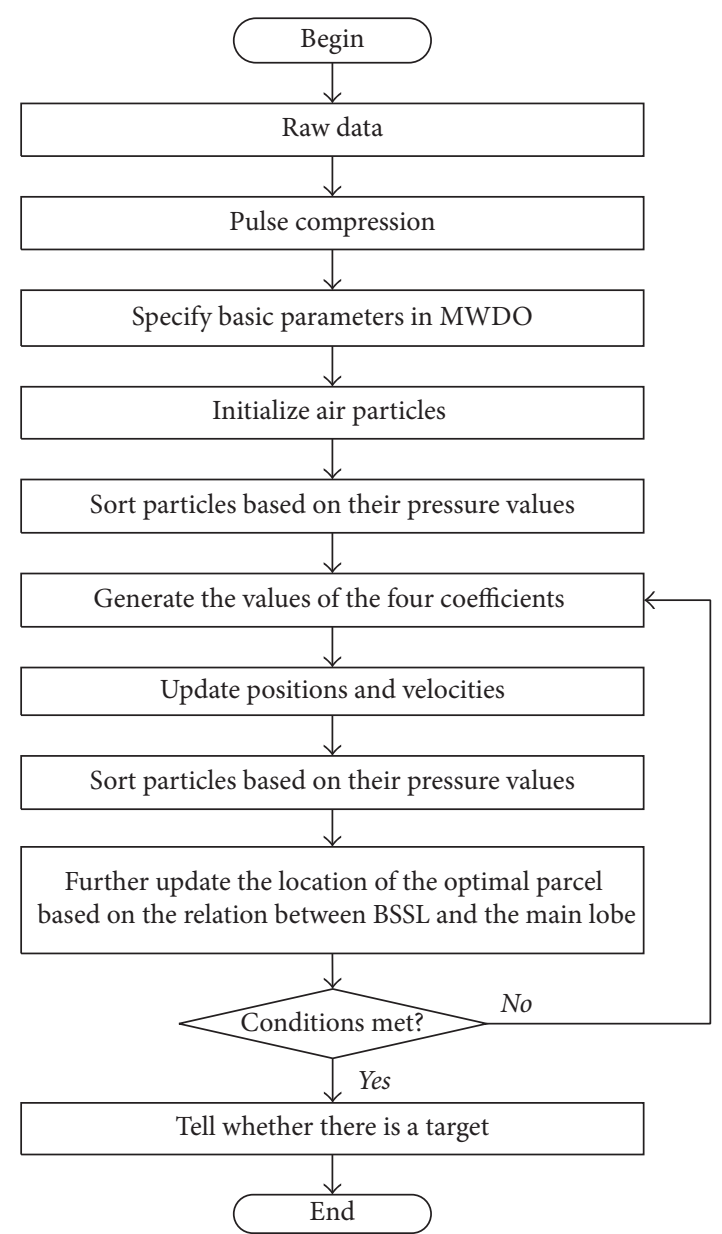

FIgURE 2: Flow chart of the target detection method via BMWDO.

Step 3. Further update $\mathbf{X}_{\text {opt }}(k)$ by the relations between BSSL and the main lobe to avoid local convergence to BSSL:

$$
\mathbf{X}_{\text {opt }}(k)=\arg \max _{\mathbf{X}_{\mathrm{opt}}}\left|G\left(\mathbf{X}_{\mathrm{opt}}(k ; q)\right)\right| \text {, }
$$

where $\mathbf{X}_{\text {opt }}(k ; q)=\left(\widehat{\alpha}_{0}, \widehat{\alpha}_{1}+q v_{b}, \widehat{\alpha}_{2}, \ldots, \widehat{\alpha}_{P-1}\right)$. As analyzed in Section 2, the amplitude of BSSL is always smaller than that of the target's main lobe; thus (25) is reasonable.

Step 4. Repeat Step 2 to Step 3 until one of the following two conditions is met:

(1) $\left|G\left(\mathbf{X}_{\text {opt }}(k)\right)\right|>\gamma$ and $k \leq k_{\max }$.

(2) $\left|G\left(\mathbf{X}_{\text {opt }}(k)\right)\right| \leq \gamma$ and $k>k_{\max }$.

The parameter $\gamma$ is the detection threshold calculated from the preset false alarm probability.

It should be pointed out that when condition (1) is met, the target is detected and when condition (2) is met, it means that there is no target. 


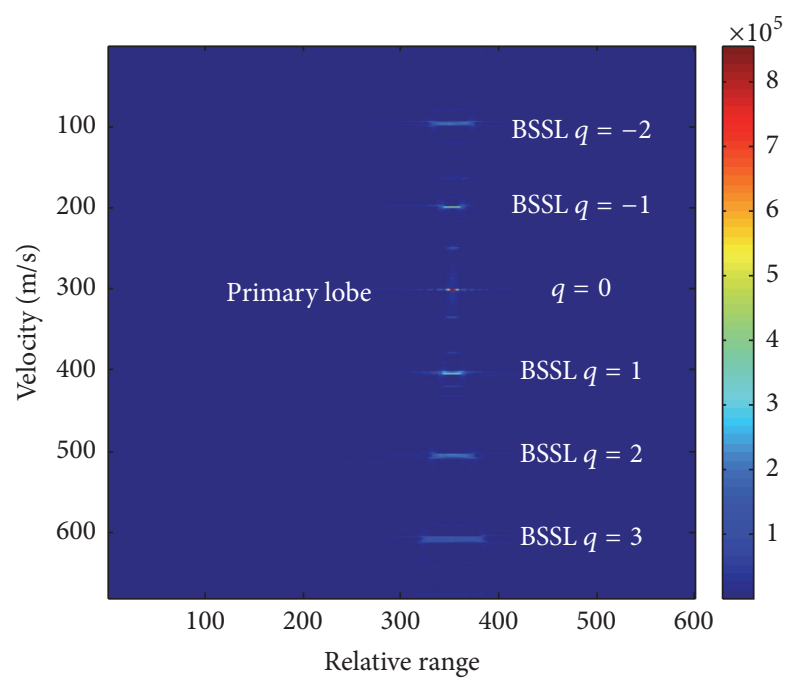

FIGURE 3: Range and velocity slice of GRFT.

\section{Numerical Results}

In this section, several numerical experiments are provided to demonstrate the effectiveness of the proposed fast implementation method of GRFT. The BSSL phenomenon is firstly verified and then BMWDO and BPSO are compared in convergence performance. The detection performances of the two fast implementation methods as well as the traditional GRFT, RFT, and moving target detection (MTD) are compared. The running time of the traditional GRFT, the BPSObased GRFT, and the BMWDO-based GRFT is also provided.

4.1. BSSL Phenomenon. In this part, we suppose that the radar pulse repetition interval $T_{r}=0.01 \mathrm{~s}$, the bandwidth $B=$ $15 \mathrm{MHz}$, the carrier frequency $f_{c}=150 \mathrm{MHz}$, and the sample frequency $f_{s}=2 B$. The blind speed $v_{b}$ can be calculated as $100 \mathrm{~m} / \mathrm{s}$. Suppose that the actual velocity is $300 \mathrm{~m} / \mathrm{s}$ and the searching range of velocity is $[1 \mathrm{~m} / \mathrm{s}, 680 \mathrm{~m} / \mathrm{s}]$. The range and velocity slice of GRFT is shown in Figure 3.

From Figure 3 we can intuitively see that, with the increase of $|q|$, the supporting area of BSSL becomes longer and the amplitude of the side lobe decreases, which corresponds to the conclusion drawn from (17). Because the amplitude of BSSL is always lower than that of the main lobe, it is reasonable to employ (25) to help parcels jump out of local convergence to BSSL.

4.2. Convergence Performance. In the following simulations, the radar parameters listed in Table 1 are adopted. The population size $S=150$ and the maximum number of iterations $k_{\max }=3000$ are specified for BPSO and BMWDO. Suppose that the motion parameter vector is $\boldsymbol{\alpha}=[62.5 \mathrm{~km}$, $\left.160 \mathrm{~m} / \mathrm{s}, 5 \mathrm{~m} / \mathrm{s}^{2}, 2 \mathrm{~m} / \mathrm{s}^{3}\right]$, and the searching ranges of the radial range, velocity, acceleration, and jerk can be given as $[60 \mathrm{~km}, 63 \mathrm{~km}],[0 \mathrm{~m} / \mathrm{s}, 300 \mathrm{~m} / \mathrm{s}],\left[-30 \mathrm{~m} / \mathrm{s}^{2}, 30 \mathrm{~m} / \mathrm{s}^{2}\right]$, and $\left[-20 \mathrm{~m} / \mathrm{s}^{3}, 20 \mathrm{~m} / \mathrm{s}^{3}\right]$, respectively. Basic coefficients of PSO are chosen according to [24]. Figure 4 shows the mean results
TABLE 1: Simulation parameters of radar.

\begin{tabular}{lc}
\hline Carrier frequency & $1 \mathrm{GHz}$ \\
Bandwidth & $15 \mathrm{MHz}$ \\
Sample frequency & $60 \mathrm{MHz}$ \\
Pulse duration & $25 \mu \mathrm{s}$ \\
Pulse repetition frequency & $100 \mathrm{~Hz}$ \\
Pulse number & 100 \\
\hline
\end{tabular}

of 20 runs of BPSO and BMWDO. The SNR of the raw data is set to be $-10 \mathrm{~dB}$ and $-28 \mathrm{~dB}$, and convergence graphs under the two cases are shown in Figures 4(a) and 4(b), respectively.

Figure 4(a) demonstrates that when SNR is relatively high, both BPSO and BMWDO can converge to the optimal value, which means that the BSSL learning-based method can avoid local convergence to side lobes effectively. We can also discover that BPSO converges faster than BMWDO. From Figure 4(b), it can be seen that, under low SNR, neither BMWDO nor BPSO can converge to the optimal value for each run. This is because when SNR becomes too low, the target is nearly undetectable even after coherent integration and the intelligent optimization algorithms will converge to noise peaks easily. It is worth paying attention to that the mean pressure values of 20 runs of BMWDO are closer to the optimal value in Figure 4(b). This result indicates that BMWDO has greater chance to jump out of the local convergence to noise peaks compared with BPSO; in other words, BMWDO has better antinoise performance although it has slower convergence speed.

4.3. Detection Performance. The detection performances of traditional GRFT, BPSO-based GRFT, BMWDO-based GRFT, RFT, and MTD are investigated via Monte Carlo trials. The false alarm probability is set as $P_{\mathrm{fa}}=10^{-6}$. Figure 5 shows the detection probabilities of the five detectors with different motion orders. It should be noted that when the motion order equals one, the traditional GRFT degenerates to RFT. It should be pointed out that $P$ is the dimension of searching space while $P-1$ is the motion order.

Figure 5 demonstrates that the detection performances of the BMWDO-based GRFT are always better than that of the BPSO-based GRFT under different SNR values or different motion orders. Especially when the motion order equals 1, the detection performance of BMWDO-based GRFT nearly reaches the ideal performance of GRFT. Making comparisons between Figures 5(a), 5(b), and 5(c), we can notice that, with the increase of motion orders, the detection performance of BMWDO-based GRFT decreases. The reason is that when motion order increases, the dimension of the searching space also increases, and the difficulty in finding the optimal solution increases too.

It is not difficult to discover that the decline of the detection probability is not obvious when motion order changes from 2 to 3 . This is because the high-order motion parameters have much lower influence on ARU and DFM compared with low-order parameters. When applying BMWDO or BPSO, 


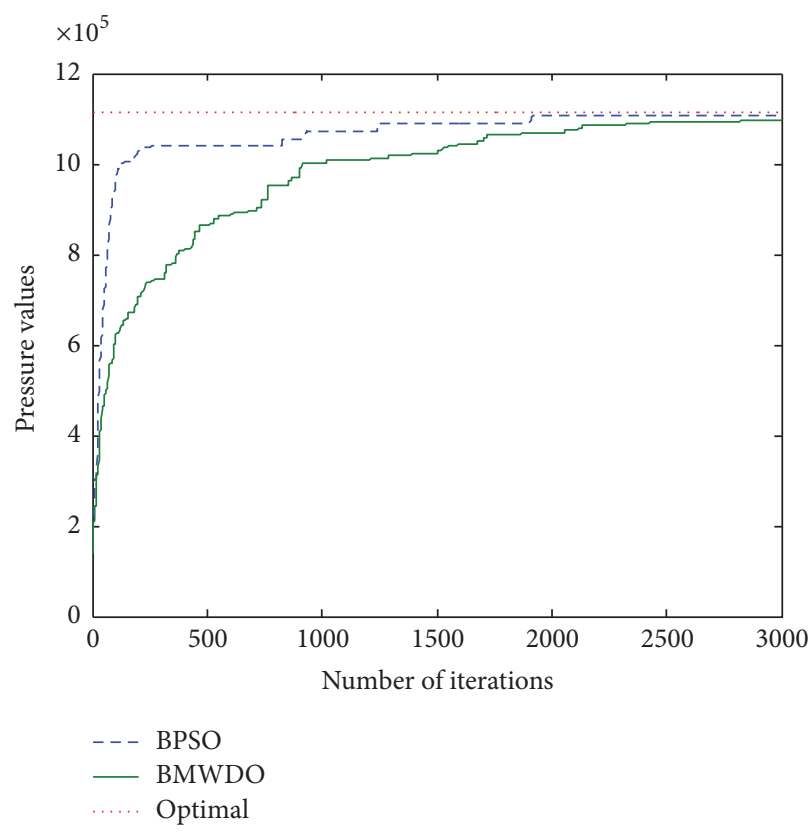

(a)

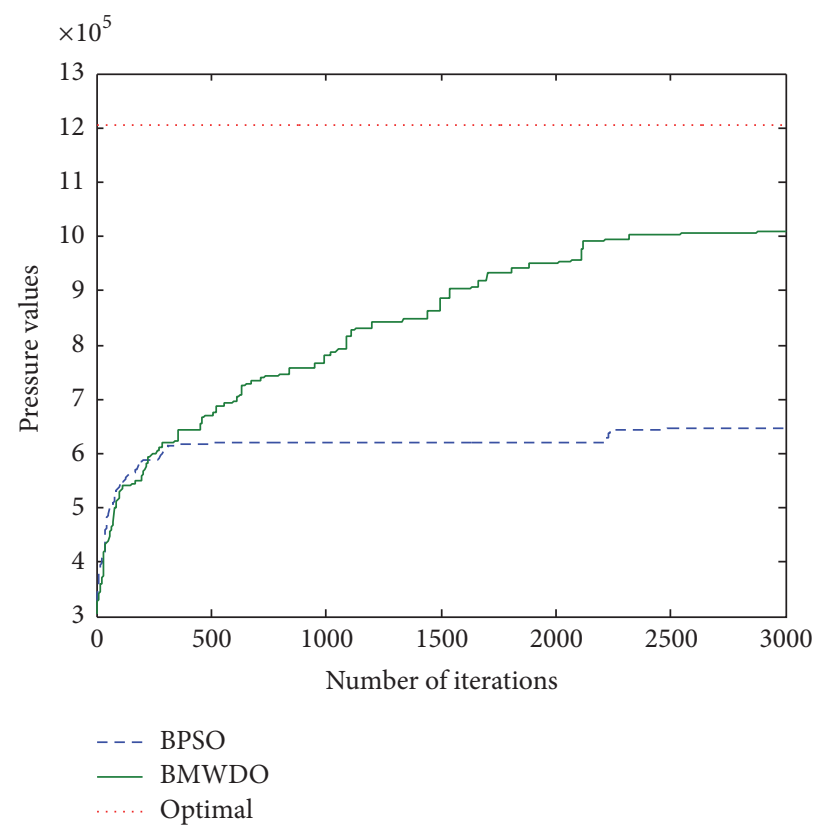

(b)

FIGURE 4: Average convergence graphs of BPSO and BMWDO with 20 runs under different SNR (before pulse compression). (a) SNR = $-10 \mathrm{~dB}$. (b) $\mathrm{SNR}=-28 \mathrm{~dB}$.

the low-order motion parameters are dominant in deciding the movement trend of air parcels. Thus, based on the radar parameters adopted in this paper, it is possible to neglect the higher motion parameters and set $P=4$ for BMWDO in the case that the motion order is greater than or equal to 3. Suppose that the target might have higher motion parameters, such as $\alpha_{4}=20 \mathrm{~m} / \mathrm{s}^{4}, \alpha_{5}=20 \mathrm{~m} / \mathrm{s}^{5}$, and $\alpha_{6}=20 \mathrm{~m} / \mathrm{s}^{6}$. With $P=4$ preset in BMWDO, the detection probabilities of BMWDO-based GRFT with motion orders no less than 3 under different SNR are shown in Figure 6. Due to the randomness of BMWDO and the statistical error, the detection probabilities fluctuate with motion orders, but we can conclude that there is no apparent detection performance loss when choosing $P=4$ for BMWDO in the case that $P>4$.

4.4. Computational Cost. In this simulation, the computational costs of the traditional GRFT, the BPSO-based GRFT, and the BMWDO-based GRFT are investigated. The searching ranges of parameters in the traditional GRFT are the same as that in BMWDO and the searching interval of each parameter can be determined according to [14]. Denote the number of range cells, pulse numbers, and motion parameter $\alpha_{p}(p=1,2,3, \ldots, P-1)$ by $N_{0}, M$, and $N_{p}$, respectively. Then the computational complexity is $O\left(\prod_{p=1}^{P-1} N_{0} M N_{p}\right)$ for the traditional GRFT. In this subsection, BPSO and BMWDO are terminated when the number of iterations reaches $k_{\max }$. In fact, when condition (1) in Step 4 is met, the two algorithms can be terminated earlier. The running time of BPSO-based GRFT and BMWDO-based GRFT can be experimented accurately. But, for the traditional ergodic-search GRFT, it is difficult to experiment its running time accurately because it is too time consuming. By analyzing, we found that the computational complexity of the traditional GRFT mainly comes from the nested loop; thus, we can calculate the running time of the traditional GRFT by multiplying the loop times with the time required for a loop. The running time of the three algorithms is shown in Figure 7. It is worth noting that when GRFT is applied in engineering, the parallel computing will be used, and the running time of traditional GRFT will not be so horrible but still will be much longer than that of the parallel computed fast implementation methods.

Figure 7 shows that the computational complexity of BPSO-based GRFT and BMWDO-based GRFT is far less than that of the ergodic-search GRFT. The running time of BMWDO is slightly longer than that of BPSO, which is acceptable. With the increase of motion order, the running time of the traditional GRFT grows nearly exponentially while the running time of BPSO and BMWDO stays stable. Therefore, efficiency and physical realizability of the proposed BMWDO-based GRFT can be validated.

\section{Conclusions}

In this paper, we propose a fast implementation method for GRFT to reduce the computational burden, namely, BMWDO. By applying BMWDO, a large number of unnecessary searching paths can be eliminated and the local convergence to BSSL can be avoided. Several numerical experiments are provided to analyze the performance of BMWDO in detail, including the convergence performance, the detection performance, and the computational burden. Compared with the traditional ergodic-search GRFT, the 


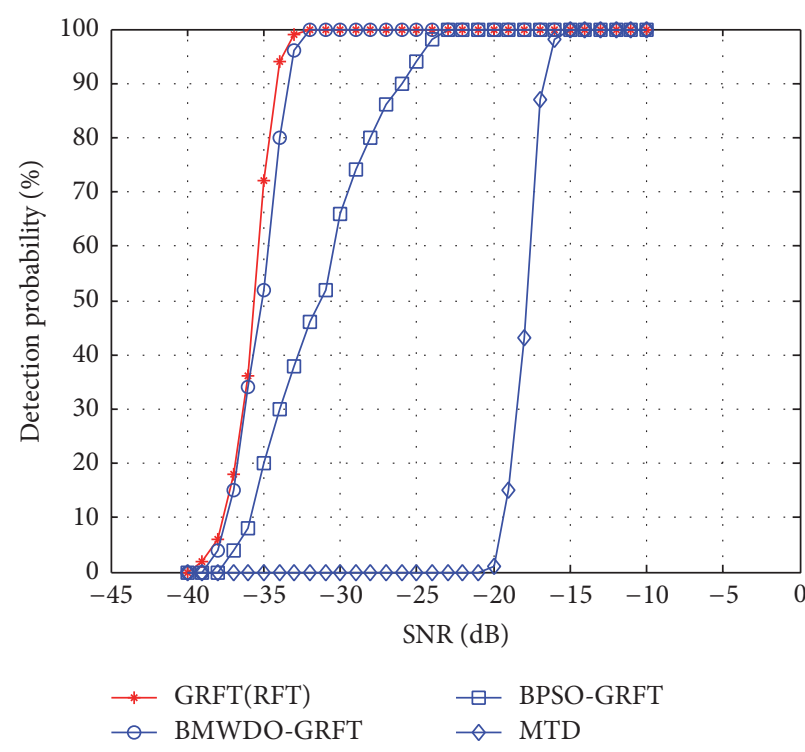

(a)

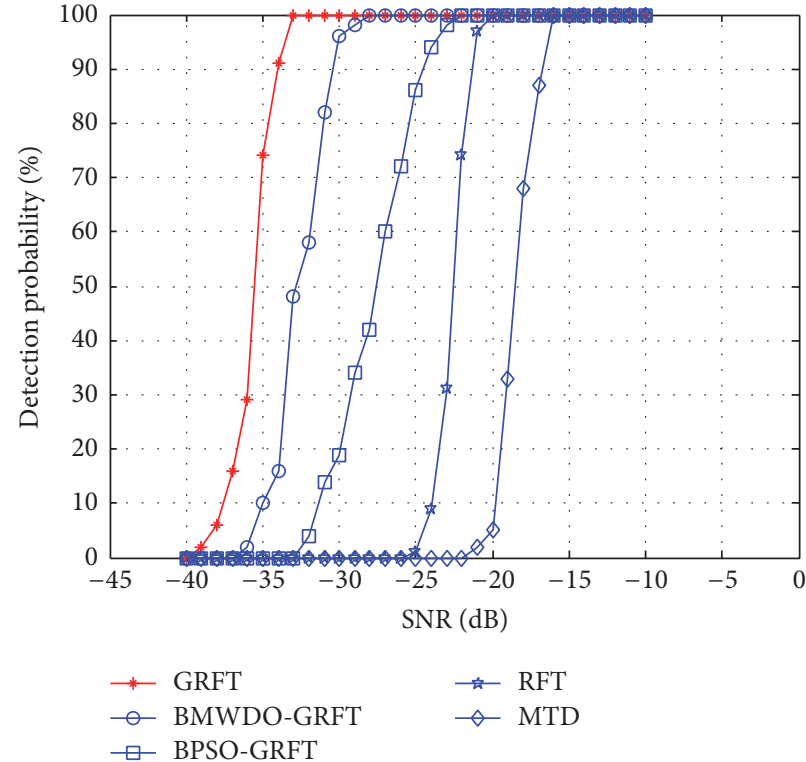

(b)

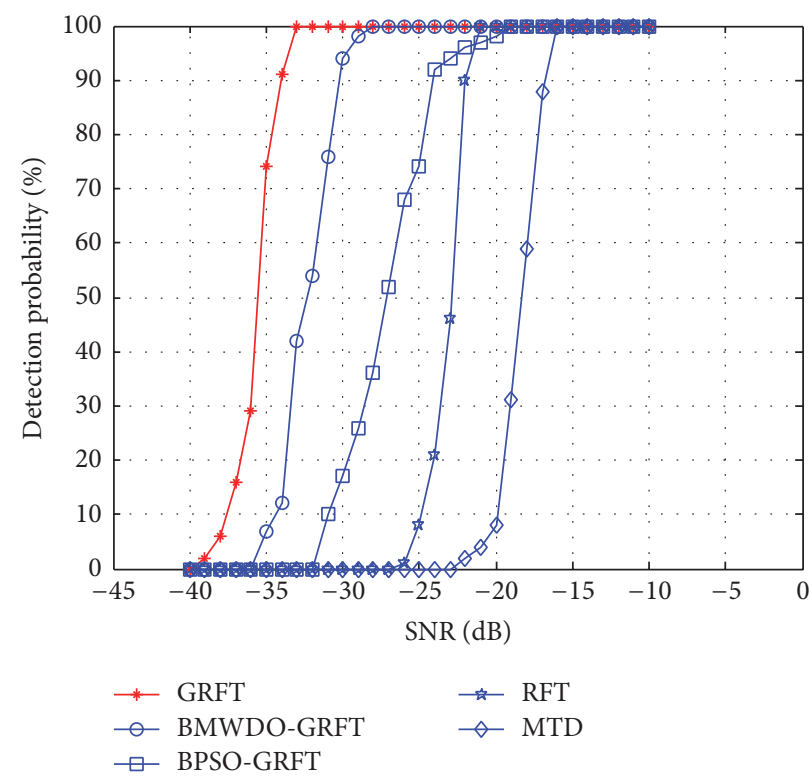

(c)

FIGURE 5: Detection performances of five different detectors with different motion orders. (a) Motion order is $1(P=2)$. (b) Motion order is $2(P=3)$. (c) Motion order is $3(P=4)$.

proposed method can realize the weak maneuvering target detection in a much more efficient way. Compared with BPSO, the BMWDO has better antinoise performance, which indicates that BMWDO has greater chance to converge to the target's main lobe in a relatively low SNR. The simulation results show that BMWDO has better detection performance and slightly longer running time compared with BPSO, which verify the effectiveness of the proposed method. At last, we should notice that although the BMWDO obviously improves the detection performance compared with BPSO, it still suffers from detection performance loss compared with the traditional GRFT. The reason is that the BMWDO is a stochastic optimization method and it cannot jump out of the convergence to noise peaks each time. Thus, our future work may further study the WDO method and combine it with other algorithms to obtain stronger antinoise performance.

\section{Competing Interests}

The authors declare that there are no competing interests regarding the publication of this paper. 


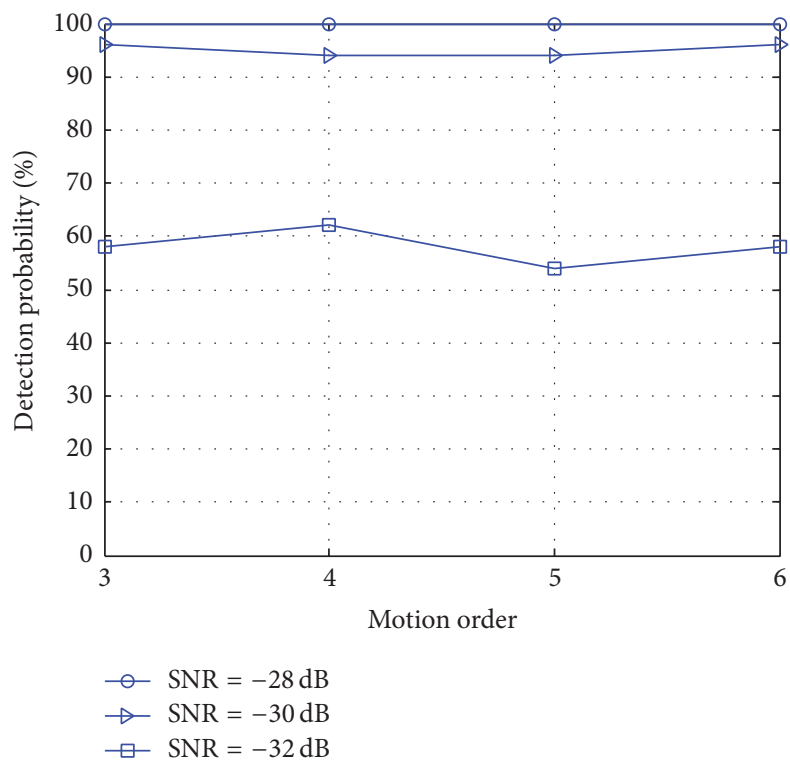

FIGURE 6: Detection probabilities of BMWDO-based GRFT with motion orders no less than $3 . P=4$ is preset when calculating BMWDO.

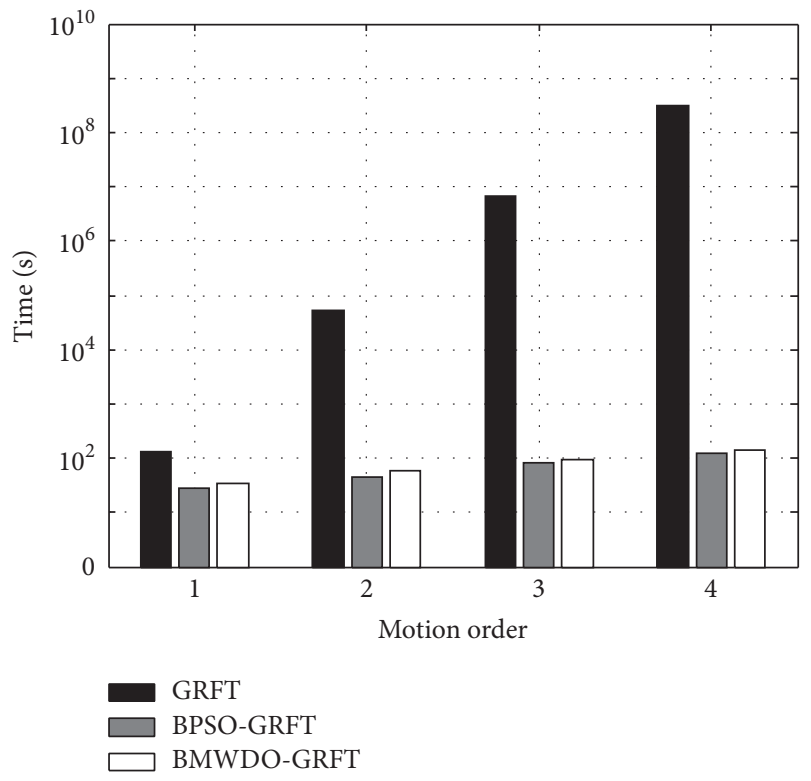

FIGURE 7: Computational cost of GRFT, BPSO-based GRFT, and the proposed BMWDO-based GRFT.

\section{Acknowledgments}

The work was supported by the National Natural Science Foundation of China (Grant no. 61201366), the Fundamental Research Funds for the Central Universities (Grant no. NS2016040), the Fundamental Research Funds for the Central Universities (Grant no. NJ20150020), and the Priority Academic Program Development of Jiangsu Higher Education Institutions.

\section{References}

[1] J. Su, M. Xing, G. Wang, and Z. Bao, "High-speed multitarget detection with narrowband radar," IET Radar, Sonar and Navigation, vol. 4, no. 4, pp. 595-603, 2010.

[2] M. I. Skolnik, Introduction to Radar System, McGraw-Hill, New York, NY, USA, 3rd edition, 2002.

[3] R. Tao, N. Zhang, and Y. Wang, "Analysing and compensating the effects of range and Doppler frequency migrations in linear frequency modulation pulse compression radar," IET Radar, Sonar and Navigation, vol. 5, no. 1, pp. 12-22, 2011.

[4] S.-S. Zhang and T. Zeng, "Weak target detection based on keystone transform," Acta Electronica Sinica, vol. 33, no. 9, pp. 1675-1678, 2005.

[5] D. Y. Zhu, Y. Li, and Z. D. Zhu, "A keystone transform without interpolation for SAR ground moving-target imaging," IEEE Geoscience and Remote Sensing Letters, vol. 4, no. 1, pp. 18-22, 2007.

[6] F. Zhou, Y.-C. Li, M.-D. Xing, and Z. Bao, "An effective approach to ground moving target imaging and motion parameter estimation for single channel SAR system," Acta Electronica Sinica, vol. 35, no. 3, pp. 543-548, 2007.

[7] X. Z. Tian, S. S. Zhang, and L. N. Pang, "Range cell migration correction for dim maneuvering target detection," in Proceedings of the IEEE Radar Conference, pp. 1247-1250, IEEE, Cincinnati, Ohio, USA, May 2014.

[8] D. Kirkland, "Imaging moving targets using the second-order keystone transform," IET Radar, Sonar and Navigation, vol. 5, no. 8, pp. 902-910, 2011.

[9] L. Kong, X. Li, G. Cui, W. Yi, and Y. Yang, "Coherent integration algorithm for a maneuvering target with high-order range migration," IEEE Transactions on Signal Processing, vol. 63, no. 17, pp. 4474-4486, 2015.

[10] J. Xu, J. Yu, Y.-N. Peng, and X.-G. Xia, "Radon-fourier transform for radar target detection, I: generalized doppler filter bank," IEEE Transactions on Aerospace and Electronic Systems, vol. 47, no. 2, pp. 1186-1202, 2011.

[11] J. Xu, J. Yu, Y.-N. Peng, and X.-G. Xia, "Radon-fourier transform for radar target detection (II): blind speed sidelobe suppression," IEEE Transactions on Aerospace and Electronic Systems, vol. 47, no. 4, pp. 2473-2489, 2011.

[12] J. Yu, J. Xu, Y.-N. Peng, and X.-G. Xia, "Radon-Fourier transform for radar target detection (III): optimality and fast implementations," IEEE Transactions on Aerospace and Electronic Systems, vol. 48, no. 2, pp. 991-1004, 2012.

[13] J. Xu, X.-G. Xia, S.-B. Peng, J. Yu, Y.-N. Peng, and L.-C. Qian, "Radar maneuvering target motion estimation based on generalized Radon-Fourier transform," IEEE Transactions on Signal Processing, vol. 60, no. 12, pp. 6190-6201, 2012.

[14] X. L. Chen, J. Guan, N. B. Liu, and Y. He, "Maneuvering target detection via Radon-fractional Fourier transform-based longtime coherent integration," IEEE Transactions on Signal Processing, vol. 62, no. 4, pp. 939-953, 2014.

[15] X. Li, G. Cui, W. Yi, and L. Kong, "Coherent integration for maneuvering target detection based on Radon-Lv's distribution," IEEE Signal Processing Letters, vol. 22, no. 9, pp. 1467-1471, 2015.

[16] X. Chen, Y. Huang, N. Liu, J. Guan, and Y. He, "Radonfractional ambiguity function-based detection method of lowobservable maneuvering target," IEEE Transactions on Aerospace and Electronic Systems, vol. 51, no. 2, pp. 815-833, 2015. 
[17] X. L. Chen, F. Q. Cai, Y. Cong, and J. Guan, "Radon-fractional Fourier transform and its application to radar maneuvering target detection," in Proceedings of the International Conference on Radar, pp. 346-350, September 2013.

[18] L.-C. Qian, J. Xu, X.-G. Xia, W.-F. Sun, T. Long, and Y.-N. Peng, "Fast implementation of generalised Radon-Fourier transform for manoeuvring radar target detection," Electronics Letters, vol. 48, no. 22, pp. 1427-1428, 2012.

[19] Z. Bayraktar, M. Komurcu, J. A. Bossard, and D. H. Werner, "The wind driven optimization technique and its application in electromagnetics," IEEE Transactions on Antennas and Propagation, vol. 61, no. 5, pp. 2745-2757, 2013.

[20] Z. Bayraktar, M. Komurcu, and D. H. Werner, "Wind Driven Optimization (WDO): a novel nature-inspired optimization algorithm and its application to electromagnetics," in Proceedings of the IEEE International Symposium Antennas and Propagation/CNC-USNC/URSI Radio Science Meeting, pp. 1-4, July 2010.

[21] R. Poli, J. Kennedy, and T. Blackwell, "Particle swarm optimization: an overview," Swarm Intelligence, vol. 1, no. 1, pp. 33-57, 2007.

[22] K. A. Penson and K. Gorska, "Exact and explicit probability densities for one-sided Levy stable distributions," Physical Review Letters, vol. 105, no. 21, Article ID 210604, pp. 1-4, 2010.

[23] Y. J. Liang and W. Chen, "A survey on computing Lévy stable distributions and a new MATLAB toolbox," Signal Processing, vol. 93, no. 1, pp. 242-251, 2013.

[24] S.-B. Peng, J. Xu, Y.-N. Peng, and J.-B. Xiang, "Parametric inverse synthetic aperture radar manoeuvring target motion compensation based on particle swarm optimiser," IET Radar, Sonar and Navigation, vol. 5, no. 3, pp. 305-314, 2011. 


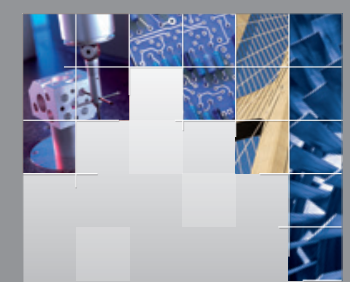

\section{Enfincering}
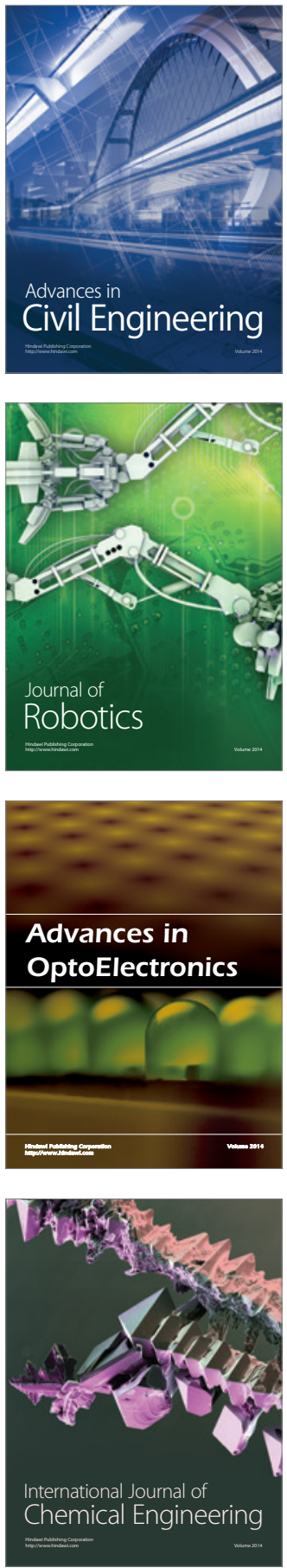

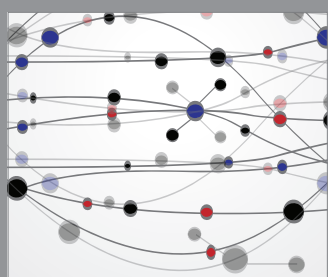

The Scientific World Journal

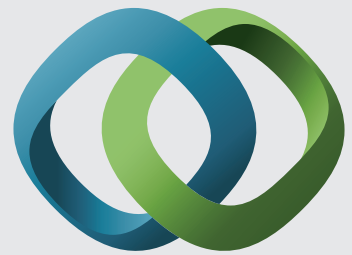

\section{Hindawi}

Submit your manuscripts at

http://www.hindawi.com
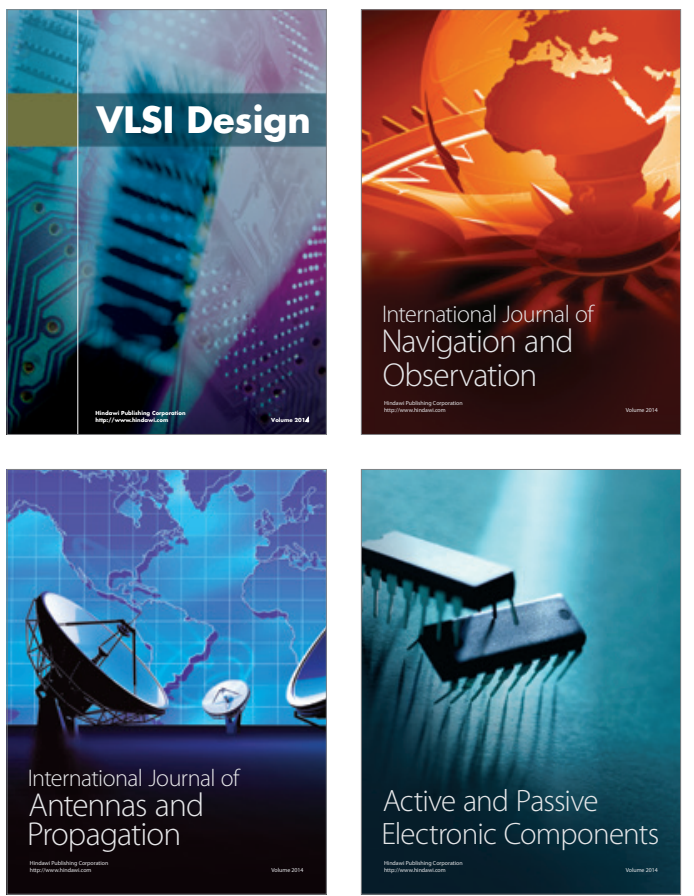
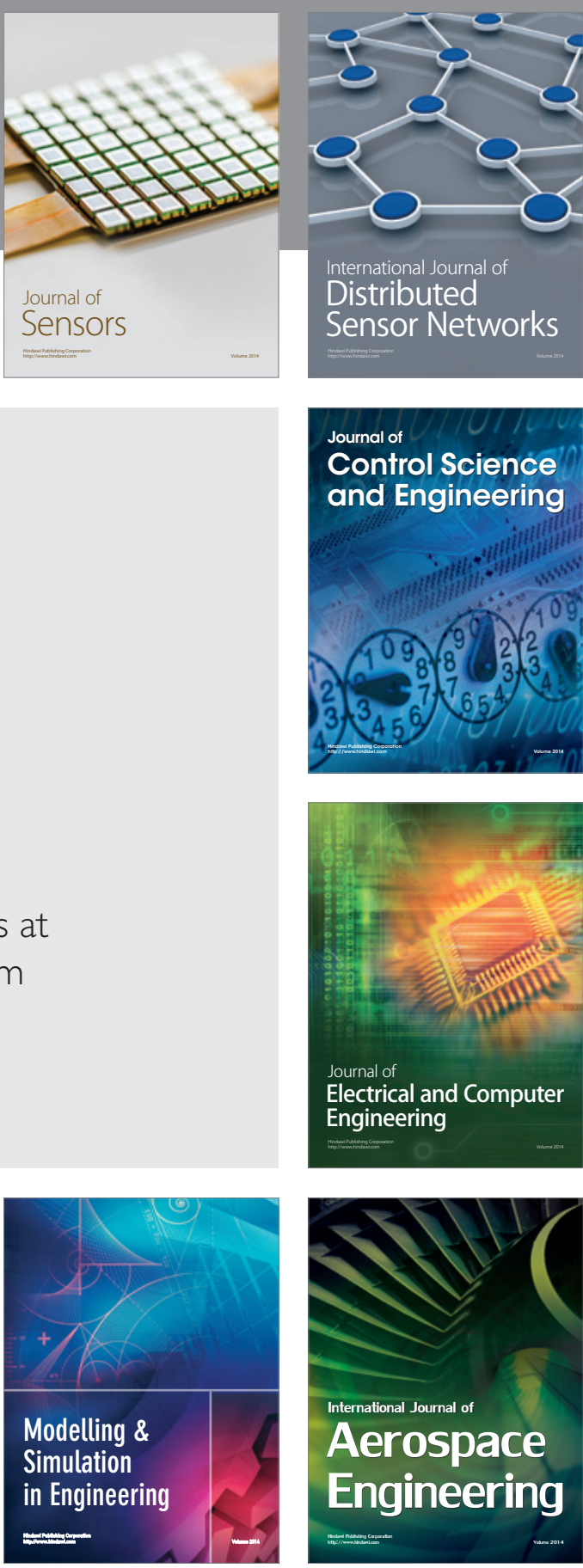

International Journal of

Distributed

Sensor Networks

Journal of

Control Science

and Engineering
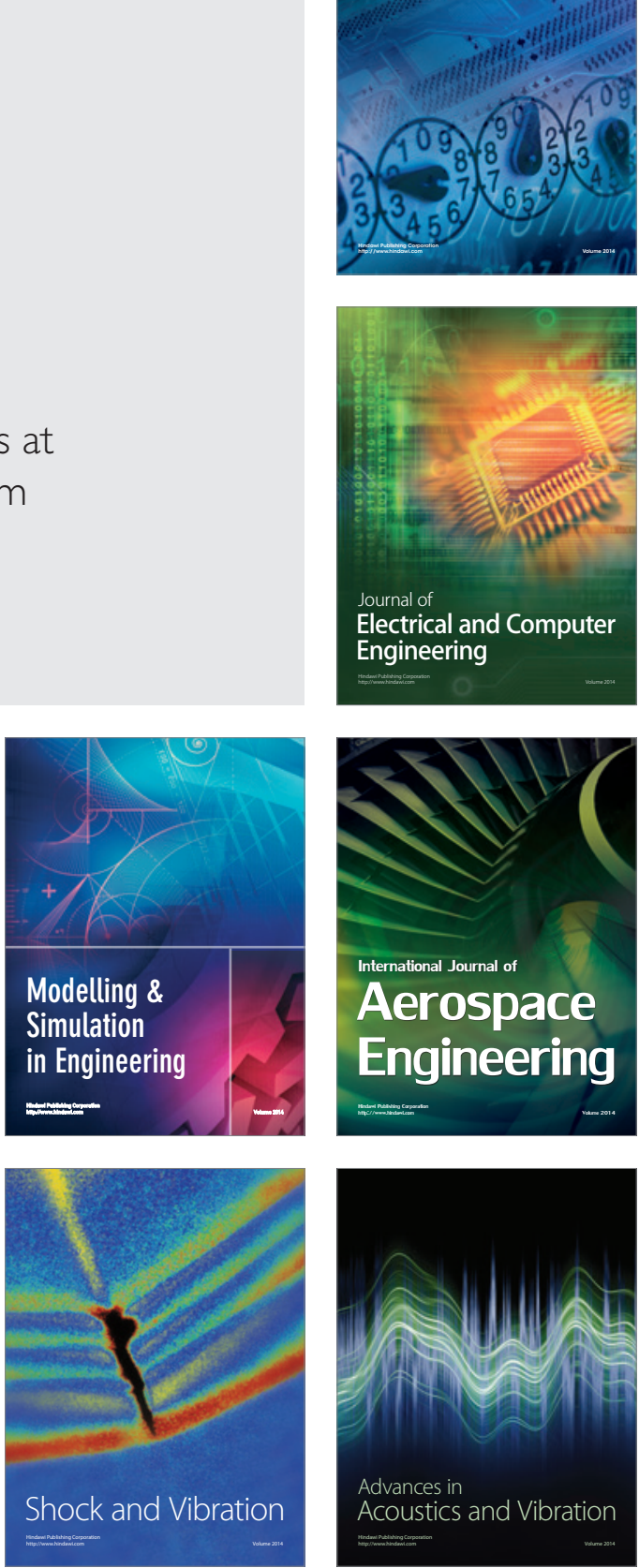